\title{
A Traffic Aware Routing Protocol for Congestion Avoidance in Content-Centric Network
}

\author{
Jung-Jae Kim ${ }^{1,}$ Min-Woo Ryu ${ }^{2 *}$, Si-Ho $\mathrm{Cha}^{3}$ and Kuk-Hyun Cho ${ }^{1}$ \\ ${ }^{1}$ Dept. of Computer Science, Kwangwoon University \\ 447-1, Wolgye-dong, Nowon-gu, Seoul 139-701, South Korea \\ $\{k j j 6929$, chokh $\} @ k w . a c . k r$
${ }^{2}$ Embedded Software Convergence Research Center, Korea Electronics Technology \\ Institute \\ \#68 Yatap-dong, Bundang-gu, Seongnam-si, Gyeonggi-do, 463-816 South Korea \\ minu@keti.re.kr \\ ${ }^{3}$ Department of Multimedia Science, Chungwoon University \\ 113, Sukgol-ro, Nam-gu, Incheon, South Korea \\ shcha@chungwoon.ac.kr
}

Content-Centric Network (CCN) is nexi generâtion internet communication technology to provide existing internet communication paradigm as content based communication for efficient use various information in the Internet. Therefore, unlike existing internet communication technology, which focus on host based communication process, all of resource is defined as contents in CCN MOreover it focuses on purpose of communication for using information. CCN communicate with between routers through broadcast flooding method in their network enviromments. This characteristic of CCN is not considering increasing netwonk traffic abou each of CCN routers. Therefore, when the requesters are rapidly increased to particalar CCN router, network congestion may be occurred by broadcast traffic. Moreoven this characteristic of CCN has low turnaround time about user request due to reducing performance of network system. To resolve this problem, this paper proposes a traffic aware routing protocol for congestion avoidance in CCN. The proposed routing protocol considers rapidly increasing traffic in the situation and establishes another routing path to favoid increasing traffic problem in CCN.

Keywords: Traffic, Content-Centric Network, Routing, Protocol, Congestion Avoidance

\section{Introduction}

Through numerous hardware technology advances, many devices were developed. And this situation is moving towards dissemination of multimedia based devices such as smart phone and tablet. According to the situation, people can connect the Internet an any-time, any-place. Theses paradigm has change of using the Internet. For example, the tool, which is used to the Internet, is moving towards from personal computer to multimedia devices. And also, using of internet information is moved from existing web page to mobile web or multimedia contents. Throughputs theses paradigm, new technology have emerged so-call Content-Centric Network (CCN). CCN is next generation internet communication technology to change existing internet communication paradigm as content based communication for

* Corresponding author 
efficient use various information in the Internet [1]. Therefore, unlike existing internet communication technology, which focus on host based communication process, all of resource is defined as contents in CCN. Moreover it focuses on purpose of communication for using information. For the change of theses communication purpose, $\mathrm{CCN}$ communicate with each $\mathrm{CCN}$ router via name based routing method and contents is saved in each CCN router. Therefore, $\mathrm{CCN}$ has different communication method existing internet communication technology.

CCN communicate through the Interest packet and the Data packet. The Interest packet is used to when requester request contents to publisher. And the Data packet is used to when publisher response contents to requester [2]. Therefore in $\mathrm{CCN}$, routing method was performed by the Interest packet and the Data packet. In there, CCN router broadcast the Interest packet to neighbor routers for finding interest contents. If $\mathrm{CCN}$ router is fined interest contents, it is create the Data packet and response it to requester via path tracing of the Interest packet. This characteristic of $\mathrm{CCN}$ is not considering increasing network traffic about each of $\mathrm{CCN}$ routers [3].

Therefore, when the requester requests are rapidly increased to particular $\mathrm{CCN}$ router, network congestion may be occurred by broadcast traffic [4]. Moreover, CCN has low network performance. This is because, $\mathrm{CCN}$ do not consider congestion avoidance in multi path routing. This problem occur low quality data service to user due to reducing network performance [5-6]. To resolve this problem this paper proposes a traffic aware routing protocol for congestion avoidance in CCW The proposed routing protocol considers rapidly increasing traffic in the situation and establishes another routing path to avoid increasing traffic problem in $\mathrm{CCN}$.

The rest of this paper is organized as follows. The existing routing protocol used in this research field is introduced in Section 2. Basicidea and overview about the proposed routing protocol is introduced in Section 3. Section 4 describes the proposed routing protocol. Section 5 presents a performance evaluation of the proposed routing protocol by comparing it with the existing CCN routing. Finally, in Section 6, conclusions are made including the future research.

\section{Related Work}

\subsection{Content-Centric Networks (CCN)}

Unlike existing internet communication method, the $\mathrm{CCN}$ communicate through name of contents instead of IP address. Therefore, in the CCN, contents name is eased to search via prefix matching due to contents name is designed as hierarchy. In addition, CCN communication through the Interest packet and Data packet. The Interest packet is used to when requester request contents to publisher and it has requested information such as content name and requester information. The Data packet is used to when publisher response contents to requester and it has contents name, information about verification, and direct data.

Figure 1 shows structure of CCN router. As shown in Figure 1, CCN router has three of structure such as Content Store (CS), Pending Interest Table (PIT), and Forwarding Information Base (FIB).

The CS has role of buffer memory in $\mathrm{CCN}$ router. It has special storage and it is caching from passed all of content through LRU caching policy about contents the inside of CCN router. The PIT tracks Interests forwarded upstream toward content source(s) so returned Data can be sent down- stream to its requester(s).

The FIB is used to forward Interest packets toward potential source(s) of matching Data. It is almost identical to an IP FIB except it allows for a list of outgoing faces rather than a single 
one. This reflects the fact that $\mathrm{CCN}$ is not restricted to forwarding on a spanning tree. It allows multiple sources for data and can query them all in parallel.

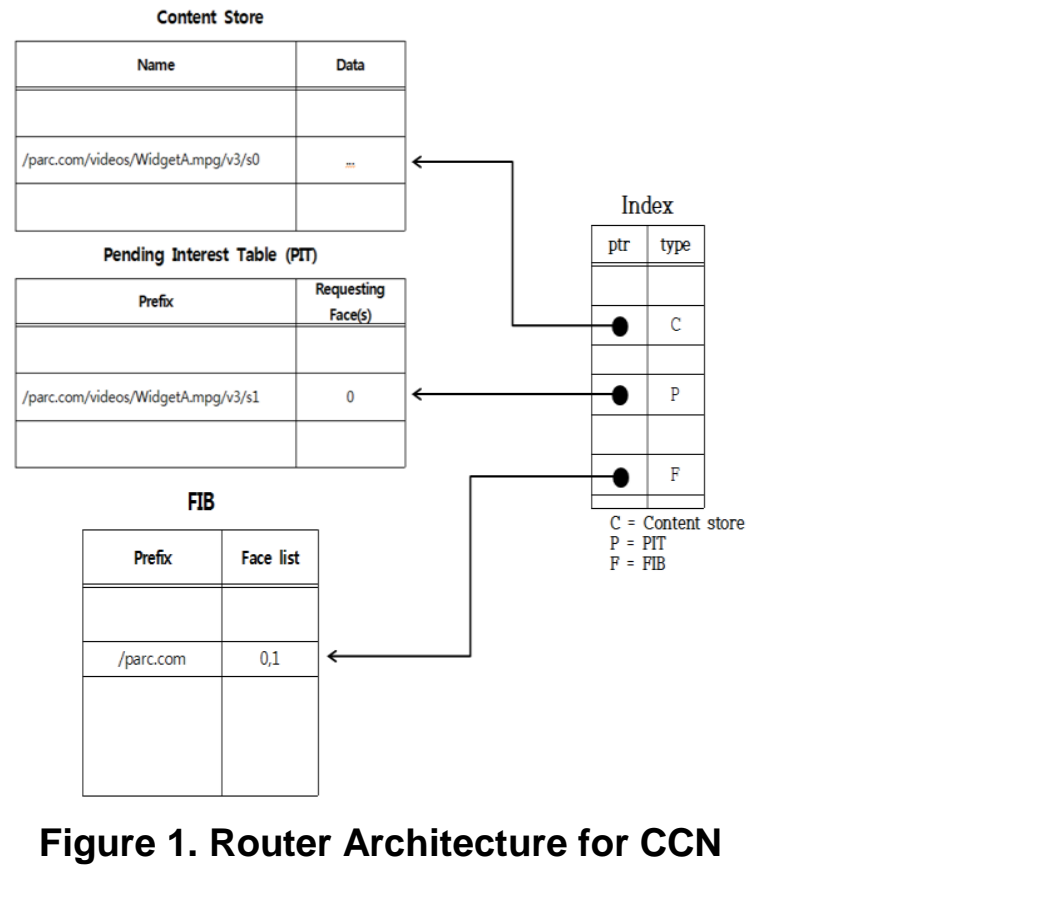

In the $\mathrm{CCN}$, which has above structure, it is not provided efficient data service due to $\mathrm{CCN}$ do not considered traffic of hodes in case of network traffic is rapidly increasing. To resolve this problem, this paper propøses a trafficuaware routing protocol for congestion avoidance in $\mathrm{CCN}$. The proposed routing protocol considers rapidly increasing traffic in the situation and establishes another fouting path to avold increasing traffic problem in CCN.

\subsection{Active Queue Management}

Active Queue Management (AQM) can execute program, which is special program in case of need process in inside CCN router when congestion is occurred. This is mean; AQM can rapidly process of congestion control through transmission path of packet than existing other congestion controls [7].

In this paper, we apply the concept of Random Early Detection (RED) [8] for measurement of traffic load among AQM process. RED was designed for sensing of congestion in packet switching network. In RED, the Control packet is discarded according average queue length, which is calculated by probability when length of queue is exceeded threshold. In other words, RED algorithm compare max threshold $\left(\max _{\mathrm{th}}\right)$ and min threshold $\left(\min _{\mathrm{th}}\right)$, which are predefined via calculating of average queue length in every packet was arrived. In RED procedure, RED has policy as follows:

- If average queue length is greater than min threshold, RED has normal condition

- If average queue length is greater than min threshold and is less than max threshold, input packets is randomly discarded.

- If average queue length is greater than max threshold, all of input packets is discarded.

Figure 2 shows drop probability about average queue length in RED. 


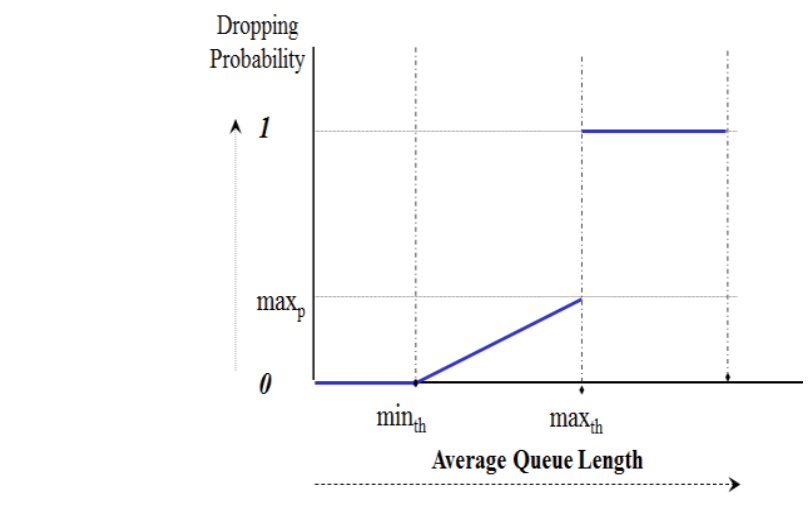

Figure 2. Packet Drop Probability of RED Algorithm

However, RED does not apply to CCN because it is different of internet communication mechanism. Therefore, in this paper, we proposed a Tralfic/Aware Routing Protocol (TARP) for congestion avoidance in CCN using concept of AQM and RED

\section{Routing Overview}

\subsection{Notations}

Unlike existing networks, the proposed TARP do not perfectly apply to RED due to it is performed in CCN environment. Therefore, we is used to only concept of RED. The table 1 list the symbols used in the proposed TARP.

\begin{tabular}{l|l}
\hline Symbols \\
\hline Aur $_{i}^{\mathrm{i}}$
\end{tabular}

\subsection{Overview}

The proposed TARP consists of 2 methods. The first method is processed the Interest packet based on average queue length by probability when average queue length is greater 
than min threshold and is less than max threshold. The second method is discarded the Interest packet when average queue length is greater than max threshold. Figure 3 show operation process of TRAP

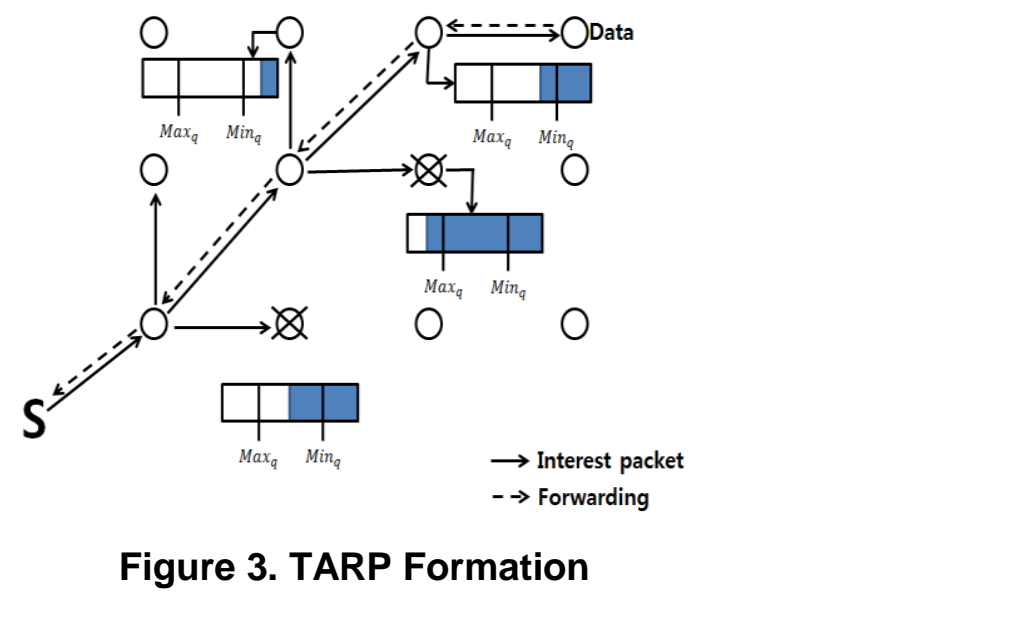

The router of TARP is defined by internal condition of router. The detail description is as follows:

- over $\mathrm{N}_{\mathrm{i}}$ : current overloaded node In other words, average queue length of $\mathrm{N}_{\mathrm{i}}$ is greater than $\max _{\text {th }}$.

- $\quad \mathrm{inN}_{\mathrm{i}}$ : overloaded node. In oher words, average queue length of $\mathrm{N}_{\mathrm{i}}$ is greater than $\max _{\text {th }}$ and is less than $\min _{\text {th }}$

- under $\mathrm{N}_{\mathrm{i}}$ : idle node. Inother words average queue length of $\mathrm{N}_{\mathrm{i}}$ is less than $\min _{\text {th }}$

All of router has at least on among above conditions. In condition of under $\mathrm{N}_{\mathrm{i}}$, there is no restriction when the Interest packet is transmitted by router and content is delivered to another router. Therefore, in case of underN, the router has same routing method as $\mathrm{CCN}$.

However, in case of oxer $\mathbb{S}_{\mathrm{i}}$, traffic condition of the router is saturation. Therefore, traffic of $\mathrm{Ni}$ is rapidly increased due to broadcasting of the Interest packet. In addition, congestion control is generated bythis situation in $\mathrm{N}_{\mathrm{i}}$. In the end, this situation leads to reducing network performance. To resolye this problem, the TARP is discarded interest packet which are received another CCN router. Thus, the router is not selected in transmission path of the Data packet.

In case of $1 \mathrm{n} \mathrm{N}_{\mathrm{i}}$, the condition is not generated reducing network performance. However, the condition may generate reducing network performance when traffic is increased. Therefore, in this situation, the TARP calculates $\mathrm{P}_{\text {drop }}^{\mathrm{i}}$ according to $\operatorname{avg}_{\mathrm{q}}^{\mathrm{i}}$ of $\mathrm{N}_{\mathrm{i}}$. And the TARP decides delay of the received Interest packet in $\mathrm{Ni}$. Through this, the TARP prevent congestion control in beforehand to exclude itself form creation of Path $\mathrm{P}_{\mathrm{i}}$.

\section{Routing Method}

In this section, we introduced routing method of the TARP. In the TARP, all of routers, which will receive the Interest packet, independently participate to establish of route according to traffic themselves. 


\subsection{Basic algorithm}

In TARP, establishing of route is started via broadcasting of the Interest packet to find contents, which are requested from request, as similar existing CCN routing. Therefore, relay nodes, which received the Interest packet, transmit the Interest packet until destination node and establish of response routing path to PIT of themselves.

After above process, the destination node traces the node which firstly received the Interest packet and transmits the Data packet including content to requester via unicasting.

In this process, the relay nodes, which receive the Interest packet, can participate to establishing of route through delay of the Interest packet or discarding of the Interest packet based on traffic condition of themselves. In other words, the relay nodes may can exclude themselves from establishing of route in case of $\mathrm{inN}_{\mathrm{i}}$ or cjtqjs This can establish routing path which can avoid nodes whereby generating of congestion in network. Moreover, this may can reduce generation of congestion in the network.

All of nodes calculate average queue length of own queue for establishing of $P a_{i}$ when the Interest packet is arrived. In this procedure, we apply exponential weighted moving average (EWMA) [9] as formula (1) for tolerance of traffic which is aryived to $\mathrm{N}_{\mathrm{i}}$ at moment when calculating of $\operatorname{avg}_{\mathrm{q}}^{\mathrm{i}}$.

$$
\operatorname{avg}_{\mathrm{q}}^{\mathrm{i}}=\left(1_{0}-{ }_{\mathrm{q}} \mathrm{avg}_{\mathrm{q} \cdot}^{\mathrm{i}}+\mathrm{W}_{\mathrm{q}} \mathrm{G}\right.
$$

In the formula (1), $\mathrm{w}_{\mathrm{q}}$ represent filter weight value of EWMA and define 0.002 as default value. And q represents new calculated length of quere. The table 2 show procedure when interest packet is arrived.

Table 2. Procedure when Interest Packet Arrives

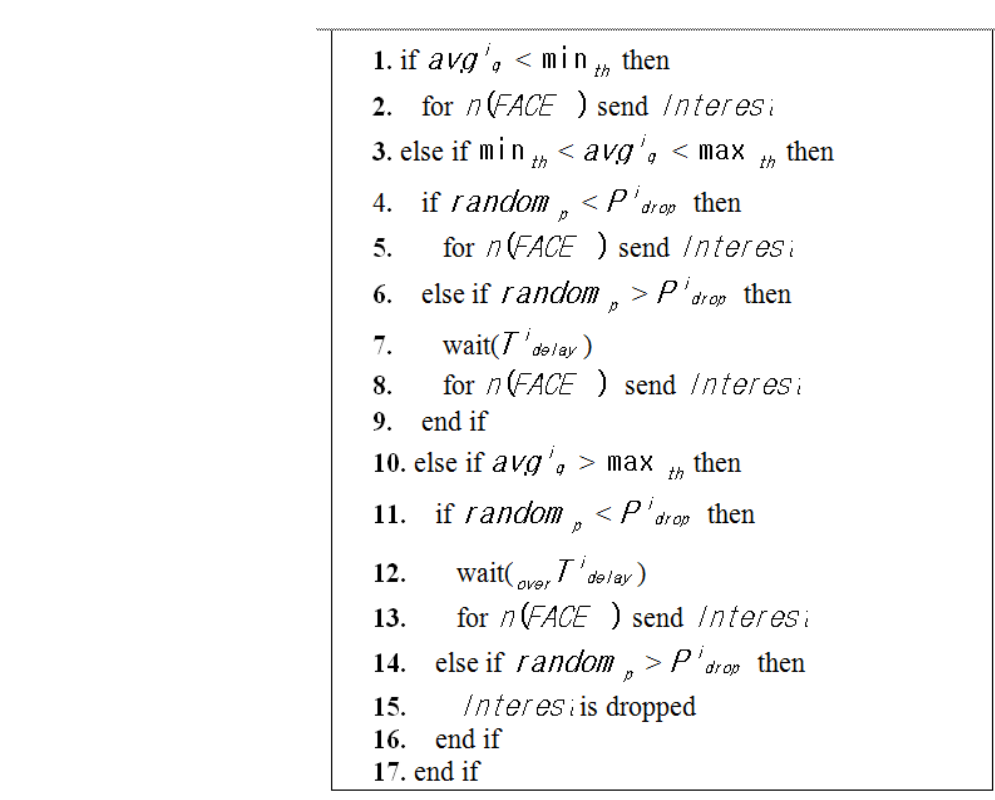

Line 2-10 describe routing method when condition of $\mathrm{Ni}$ is become underN $\mathrm{N}_{\mathrm{i}}$. And line 3-9 describe routing method when condition of $\mathrm{Ni}$ is become $\mathrm{inN}_{\mathrm{i}}$. Routing method when condition of $\mathrm{Ni}$ is become over $\mathrm{N}_{\mathrm{i}}$ is described in line 10 to 17. The each of detail description is introduced in Section 4.1, and Section 4.2 


\subsection{Method 1: in $_{\mathrm{i}}$}

The Method 1 represent processing method when $\operatorname{avg}_{q}^{1}$ is greater than $\min _{\text {th }}$ and is less than $\max _{\text {th }}$. In this condition, drop probability of the Interest packet can be obtained using formula (2).

$$
\begin{aligned}
& p_{\text {in }}^{i}=\frac{P_{\max } \times\left(\operatorname{avg}^{i} a-\min { }_{\text {th }}\right)}{\max { }_{\text {th }}-\min \text { th }_{\text {m }}} \\
& P_{\text {over }}^{i}=\frac{\left(\operatorname{avg}_{a}^{i}-\min { }_{t h}\right)}{\max { }_{\text {th }}-\min \min _{\text {th }}} \text { th }
\end{aligned}
$$

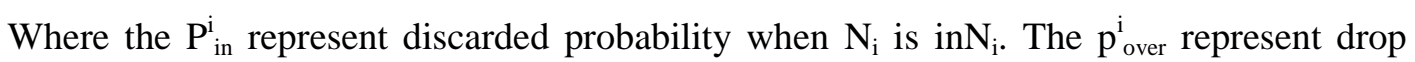
probability when $\mathrm{Ni}$ is overN $\mathrm{N}_{\mathrm{i}}$. As shown formula (2), the greater the average queue length (the larger the traffic), the greater the delay probability.

In TARP, relay nodes process the Interest packet based on queue condition of themselves using formula (2). Figure 4 shows drop probability in TARP.

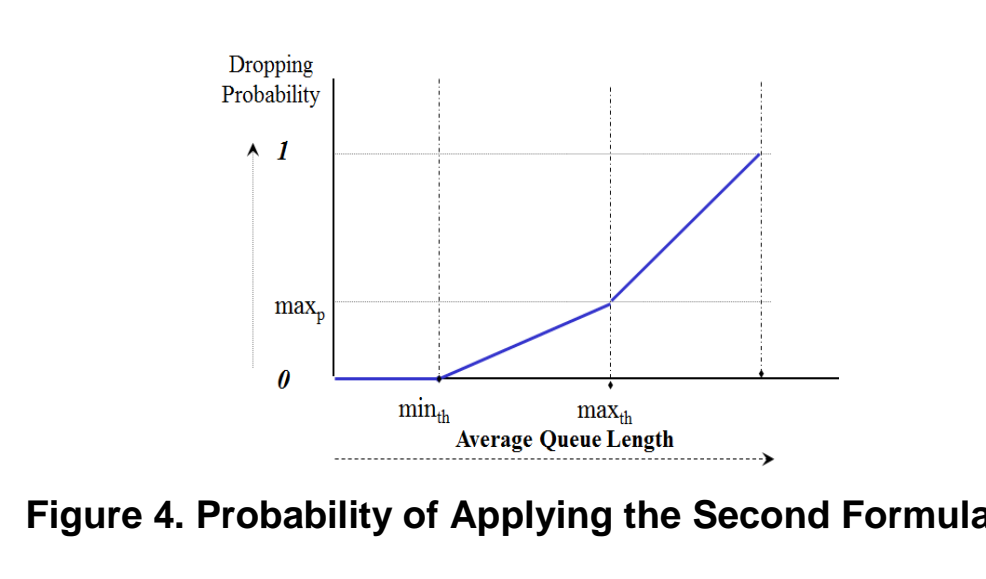

In existing RED, all of node discards the Interest packet when average queue length is exceeded max threshold.However, in this case, if any node is relay node and relay node is only one in the network, trunaround time of content has high delay due to the Interest packet drop is generated until resolving congestion. Finally, the network perforce is reduced. On the other hand, the proposed TARP can be improving survival probability of the Interest packet via formula (2).

In the proposed TARP, calculated drop probability from formula (2) is compared with Randomp. In this procedure, if calculated drop probability is less than Random $_{p}$, the TARP regards condition of $\mathrm{N}_{\mathrm{i}}$ as under $\mathrm{N}_{\mathrm{i}}$. Therefore, the Interest packet is broadcasted to another neighbor node. On the other hand, if calculated drop probability is greater than $\operatorname{Random}_{\mathrm{p}}$, the TARP delay broadcasting during predefined $\mathrm{T}_{\text {delay. }}^{\mathrm{i}}$. Therefore, overloaded node delay creation

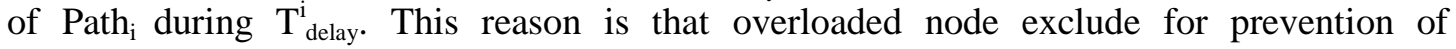
congestion in the network.

\subsection{Method 2: overN ,}

The Method 2 represents processing method when $\operatorname{avg}_{\mathrm{q}}{ }_{\mathrm{q}}$ is less than $\min _{\mathrm{th}}$. The Method 2 performs as similar of the Method 1. However, if drop probability, which is obtained $\mathrm{P}_{\text {over }}^{\mathrm{i}}$ in

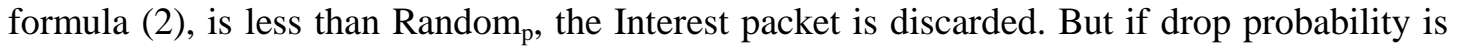


greater than Randomp, the Interest packet is delayed as equal of the Method 1. However, in case of this procedure, current node $\mathrm{N}_{\mathrm{i}}$ has traffic saturation. Therefore congestion is generated in the network when $\mathrm{N}_{\mathrm{i}}$ is selected relay node. To resolve this problem, in the Method 2, creation time of the Interest packet is forcibly increased as much as over $\mathrm{T}_{\text {delay }}^{\mathrm{i}}$ by condition of current queue. The ${ }_{\text {over }} \mathrm{T}_{\text {delay }}^{\mathrm{i}}$ is obtained using formula (3).

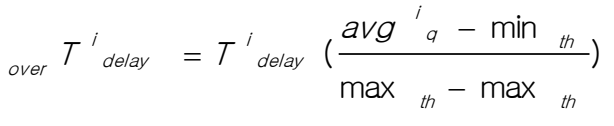

\section{Performance Evaluation}

In this section, we analyze and compare the performance of the proposed TARP and existing CCN using OPNET to prove the validity of the proposed TARP.An this performance evaluation, we considered the throughput according to input load and average turnaround time of contents.

In the simulation, each of the link bandwidth is efined 100Mbps and link delay is defined $10 \mathrm{~ms}$. And also we added traffic at every 3 -hop for making of rapidy increased traffic. Table 3 summarizes our simulation parameters.

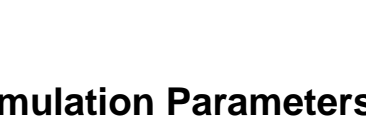

\begin{tabular}{l|l}
\hline Parameters & $100 \mathrm{Mbps}$ \\
\hline Link bandwidth & $10 \mathrm{~ms}$ \\
\hline Link delay & 96 bytes \\
\hline Control packet size & 1024 byte \\
\hline Data packet size & $20 \sim 120$ \\
\hline Number of packes &
\end{tabular}

Moreover, environment of simulation about network consists of server, user, and routers. Where server has content and the Interest packet is created by user. Finally, routers are used to transmit the Interest packet to server. In addition, we constructed mash topology for establishing of multi path and regularity of structure. Figure 5 show our network model of simulation.

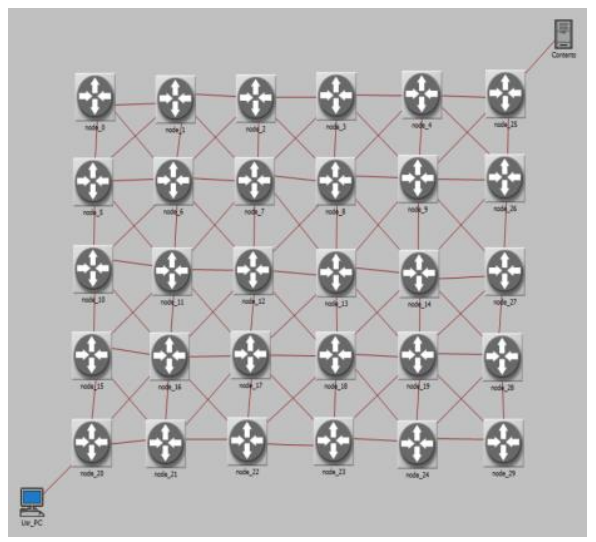

Figure 5. Network Model of Simulation 
Figure 6 shows throughput according to input load. In the figure 6, the more input load increased, the throughput decreased. When we compare CCN, the proposed TARP has higher performance than CCN. In particular, in case of existing CCN, throughput is rapidly reduced when input load is exceeded of $1000 \mathrm{Kbps}$. On the other hand, the proposed TARP is slower reduced than existing CCN. This is because the proposed TARP prevent rapidly reducing throughput via avoidance of congestion situation in previously.

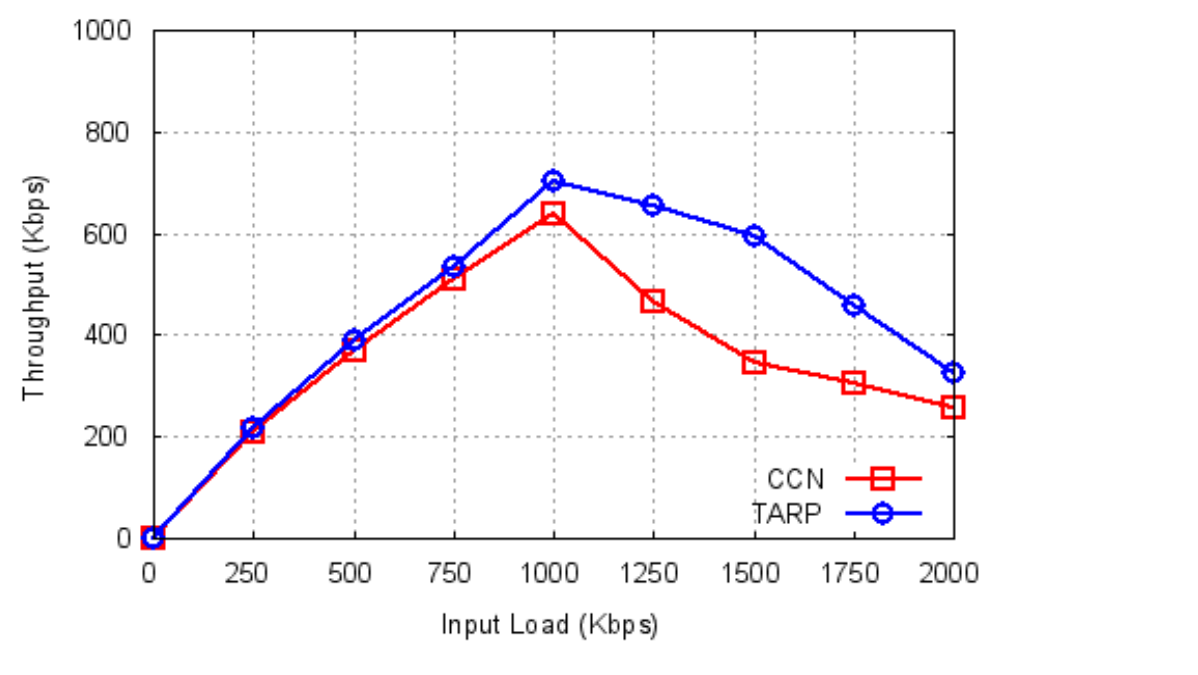

Figure 6. Throughput According to Input Load

Figure 7 shows average turnaround time of contests according to number of packets. In this figure, larger number॰of,packets led to higher average turnaround time in $\mathrm{CCN}$ and the proposed TARP. However, the average turnaround time of contents lower than existing CCN. Moreover, difference ot turnaround time between the proposed TARP and existing CCN is from minimum $20 \mathrm{nsec}$ to maximum $100 \mathrm{msec}$.

This is because the proposed TARP exclude overloaded routers in response routing path. Therefore, the proposed TARP has shorter turnaround time of contents than existing CCN.

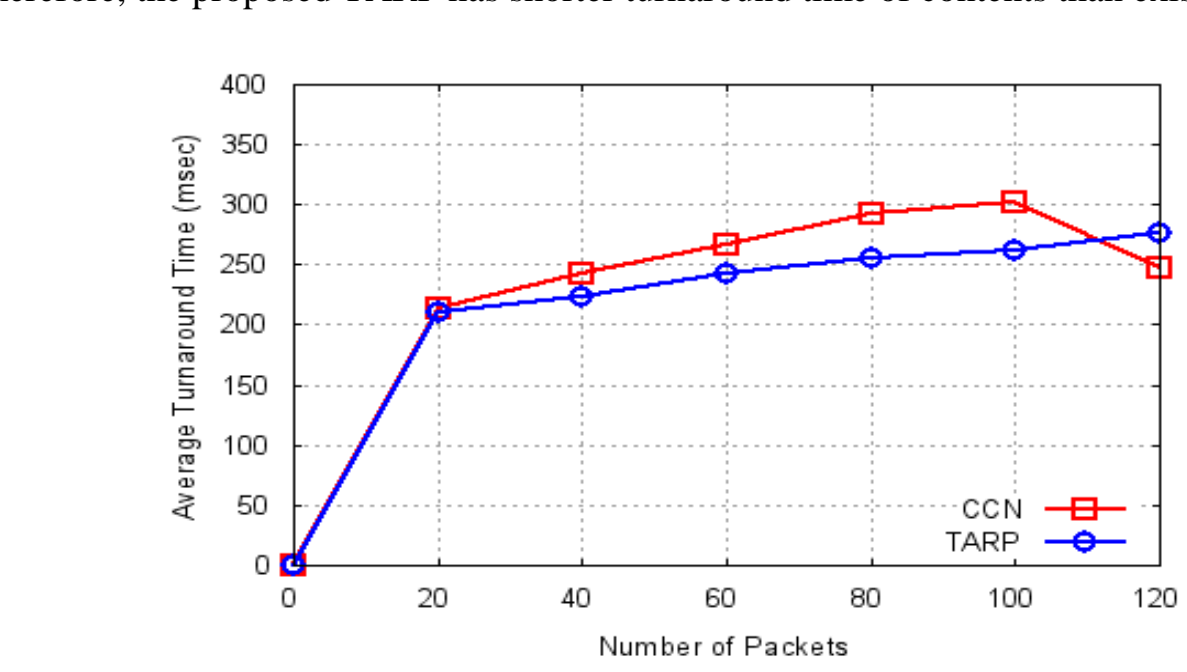

Figure 7. Average Turnaround Time of Contents 


\section{Conclusion and Future Work}

In this paper, we proposed TARP for avoidance in congestion when traffic is rapidly increased in the CCN. The proposed TARP decides participation about establishing path of route according to condition of average queue length. Moreover, we were proved the proposed TARP much better than the existing $\mathrm{CCN}$ through simulation such as throughput according to input load and average turnaround time of contents.

However, the completed Interest packet, which was used for responding of contents, still remains in the network. Therefore, increasing of traffic still exists. Future research will include distinction of the completed Interest packet and management of sole relay node according to traffic saturation.

\section{References}

[1] B. J. Lee, H. S. Jeon, and H. Y. Song "Information-Centric Networking Research Trend", Electronics and Telecommunications Trends, (2012).

[2] V. Jacobson, D. K. Smetters, J. D. Thornton, M. F. Plas\&, N. H. Briggs and R. L. Braynard, "Networking named content", In Proceedings of the $5^{\text {th }}$ ACM International Conference on Emerging Networking Experiments and Technologies, (2009), pp. 1-12.

[3] L. Wang, A. K. M. Mahmudul Hoque, C. Yi, A. Alyyan and B. Zhang, "OSPFN: An OSPF Based Routing Protocol for Named Data Networking," Technical Report NDN Technieal Report NDN-2012-13, July (2012).

[4] C. Li, W. Liu and K. Okamura, "A Greedy and Colony Forwarding Algorithm for Named Data Networking", in Proceedings of the APAN - Network Research Workshop (2012).

[5] J. Moy, "OSPF version 2", RFC 2178, (1998)

[6] J. Moy, "OSPF protocol analysis", Nefwopk Working Group, (1991) July.

[7] G. Thiruchelvi and J. Raja, "A Sturvey on Active Queue)Management Mechanisms", IJCSNS International Journal of Computer Science and Network Security, vol, 8, no. 12, (2008) December.

[8] S. Floyd and V. Jacobson, "Random Early Detection Gateways for Congestion Avoidance", IEEE/ACM Transactions on Networking, (1993) August.

[9] J. Stuart Hunter, “The Exponentially Weighted Moving Average”, Journal of Qualit Technology, vol. 18, no. 4, (1986) October.
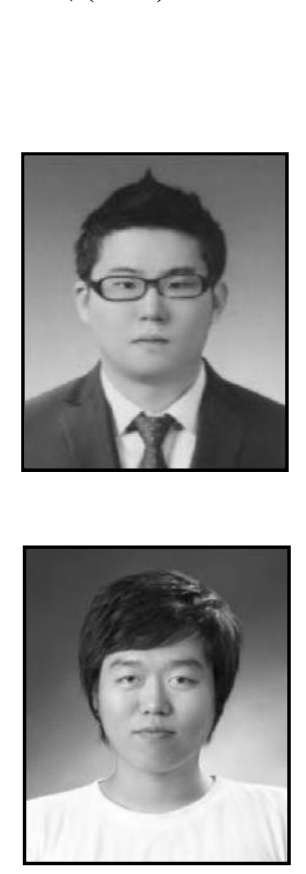

Min-Woo Ryu, he received his B.S. in Internet Information Processing from Yeoju Institute of Technology, Korea in 2007, and his M.S. and Ph.D in Computer Science from Kwangwoon University, Seoul, South Korea, in 2009 and 2012, respectively. He worked as a senior researcher in the Convergence Emerging Industries R\&D Division at the Korea Electronics Technology Institute (KETI), Seongnam, South Korea. His research interests include Vehicle Ad Hoc Networks, Informationcentric Networks, Internet of Things, and Semantic based Service. 


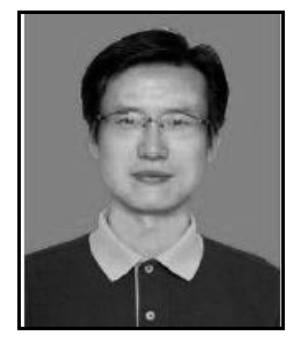

Si-Ho Cha, he received his B.S. degree in Computer Science from Sunchon National University, Sunchon, Korea, in 1995, and his M.S. and $\mathrm{Ph} . \mathrm{D}$. degrees in Computer Science from Kwangwoon University, Seoul, Korea, in 1997 and 2004, respectively. From 1997 to 2000, he worked as a senior researcher at Daewoo Telecom and he also worked as a researchin-charge at Wareplus in Korea from 2004 to 2005. He is now an Assistant Professor in the Department of Multimedia Science, Chungwoon University, Incheon, Korea. His research interests include network management, vehicular ad hoc networks, and wireless sensor networks.

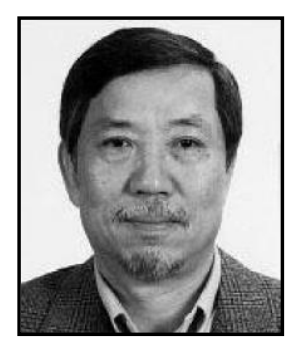

Kuk-Hyun Cho, he redeived his B.S. degree in Electronic Engineering from Hanyang University, Seoul, Korea in 1977 and his M.S. and Ph.D. degrees in Electronic Engineering from Tohoku University, Sendai, Japan in 1981 and 1984, respectively. Since 1984, he has been a Professor in the Department of Computer Science and Engineering, Kwangwoon Universily, Seôl, Korea. From 1998 to 2000, he was President of Open Standards and Internet Association (OSIA). His research interests include network and service management, wireless sensor networks, and vehicular ad hoc networks.

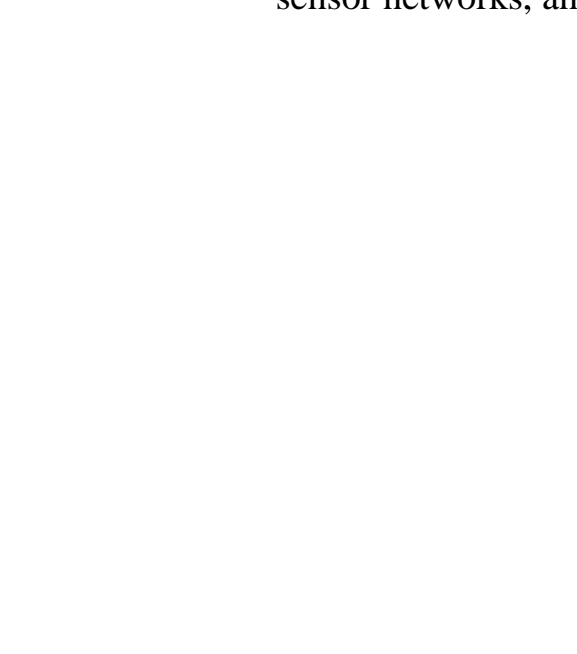


International Journal of Multimedia and Ubiquitous Engineering Vol.9, No.9 (2014)

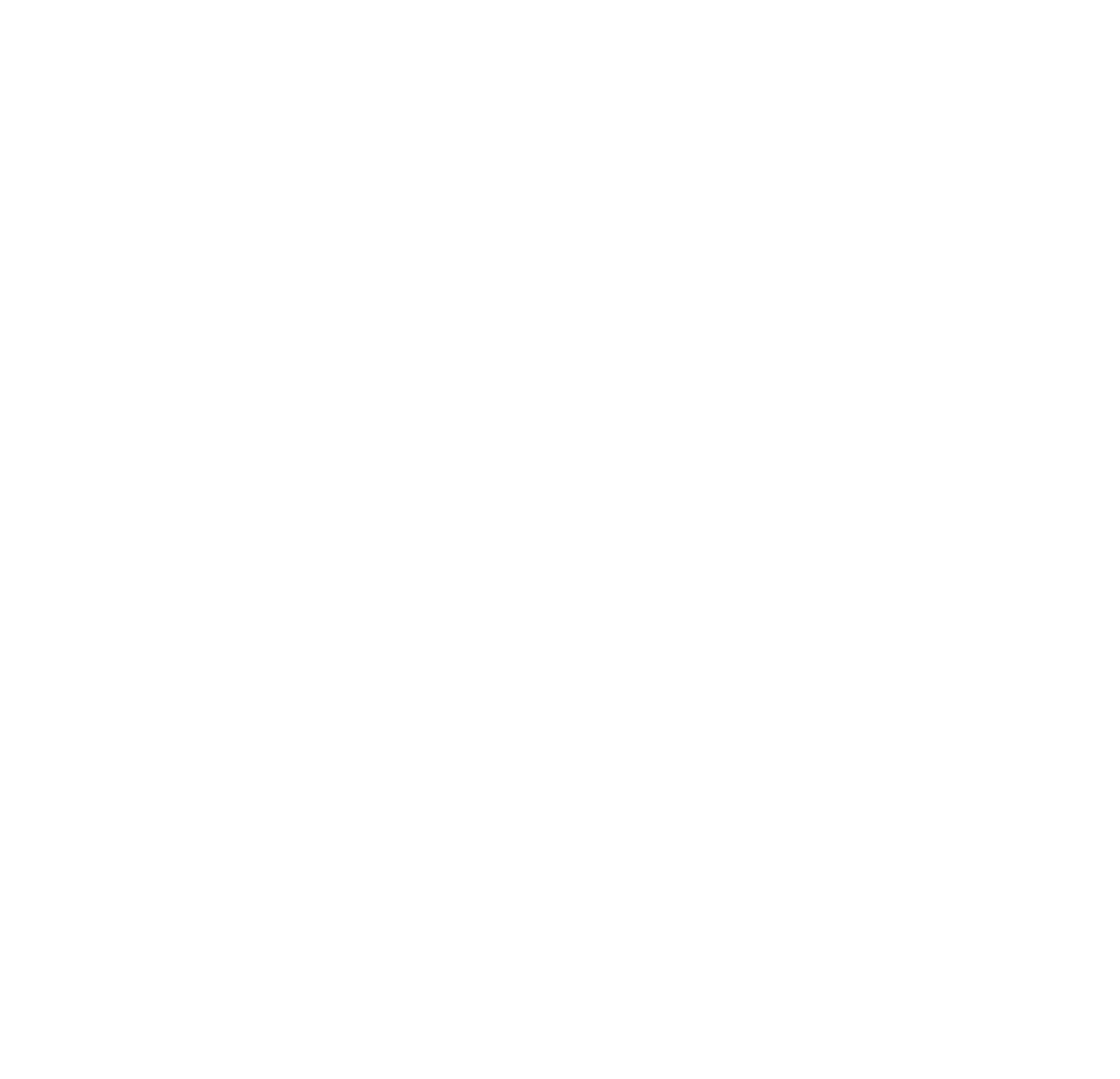

